\title{
Phytoprotection
}

\section{Avancée de Diabrotica virgifera virgifera [Coleoptera : Chrysomelidae] dans les champs de maïs au Québec et collecte dans le soja à Ottawa, Ontario Advance of Diabrotica virgifera virgifera [Coleoptera : Chrysomelidae] in corn fields in Quebec and sampling in soybean plants in Ottawa, Ontario}

\author{
F. Meloche, P. Filion, G. Tremblay et L. LeSage
}

Volume 82, numéro 1, 2001

URI : https://id.erudit.org/iderudit/706213ar

DOI : https://doi.org/10.7202/706213ar

Aller au sommaire du numéro

Éditeur(s)

Société de protection des plantes du Québec (SPPQ)l

ISSN

0031-9511 (imprimé)

1710-1603 (numérique)

Découvrir la revue

Citer cet article

Meloche, F., Filion, P., Tremblay, G. \& LeSage, L. (2001). Avancée de Diabrotica virgifera virgifera [Coleoptera : Chrysomelidae] dans les champs de maïs au

Québec et collecte dans le soja à Ottawa, Ontario. Phytoprotection, 82(1), 35-38. https://doi.org/10.7202/706213ar
Résumé de l'article

La chrysomèle des racines du maïs de l'Ouest, Diabrotica virgifera virgifera, a été trouvée au Québec en septembre 2000 dans la région de la Montérégie. Ceci constitue une extension vers le nord de son aire de répartition. De plus, à Ottawa, quelques individus se sont développés à partir du soja. Ceci constitue la première mention de développement de cet insecte sur du soja au Canada. 


\title{
Avancée de Diabrotica virgifera virgifera [Coleopte- ra : Chrysomelidae] dans les champs de maïs au Québec et collecte dans le soja à Ottawa, Ontario
}

\author{
Francois Meloche ${ }^{1}$, Pierre Filion ${ }^{2}$, Gilles Tremblay ${ }^{3}$ et Lau- \\ rent LeSage ${ }^{1}$
}

Reçu 2001-01-19; accepté 2001-05-11

PHYTOPROTECTION 82 : 35-38

La chrysomèle des racines du maïs de l'Ouest, Diabrotica virgifera virgifera, a été trouvée au Québec en septembre 2000 dans la région de la Montérégie. Ceci constitue une extension vers le nord de son aire de répartition. De plus, à Ottawa, quelques individus se sont développés à partir du soja. Ceci constitue la première mention de développement de cet insecte sur du soja au Canada.

[Advance of Diabrotica virgifera virgifera [Coleoptera : Chrysomelidae] in corn fields in Quebec and sampling in soybean plants in Ottawa, Ontario]

The western corn rootworm, Diabrotica virgifera virgifera, was found in the Monteregie region in the province of Quebec in September 2000. This finding constitutes a northern extension of the species distribution. Moreover, at Ottawa, some specimens were found developing from soybean plants. This constitutes the first mention of development of this insect on soybean in Canada.

Trois espèces de chrysomèles des racines du maïs, la chrysomèle des racines du nord, Diabrotica barberi (Smith et Lawrence), la chrysomèle des racines de l'ouest, Diabrotica virgifera virgifera LeConte et la chrysomèle des racines du sud, Diabrotica undecimpunctata howardi Barber sont présentes au Canada. Diabrotica barberi (sub longicornis) a été rapportée pour la première fois au Québec en 1975 (O'C. Guibord 1976) et est signalée régulièrement depuis $(\mathrm{Hu}$ don et al. 1984). Diabrotica u. howardi est observée de I'Alberta à la Nouvelle-
Écosse, alors que D. v. virgifera est présente en Ontario uniquement (Campbell et al. 1989). La présente communication concerne surtout $D$. $v$. virgifera chez laquelle nous avons observé une nette avancée dans l'est canadien.

En 1999, des observations chez 18 producteurs de maïs-grain répartis dans la Montérégie ouest ainsi que des échantillonnages à l'aide de filets-fauchoirs dans la Montérégie est (Saint-Hyacinthe, Saint-Pie-de-Bagot, Saint-Damase

1. Centre de Recherche de I'Est sur les Céréales et Oléagineux, Ottawa, Ontario, Canada K1A 0C6; courriel : melochefc@em.agr.ca. Contribution nº : 011619

2. Ministère de l'Agriculture, des Pêcheries et de l'Alimentation du Québec, Sainte-Martine, Québec, Canada JoS 1V0

3. Centre de recherche sur les grains, Saint-Bruno-de-Montarville, Québec, Canada J3V 4P6 
et Saint-Liboire) ont confirmé la présence de $D$. barberi et de quelques spécimens de $D$. $u$. howardi dans les cultures de luzerne, trèfle et de soja, mais aucun spécimen de $D$. $v$. virgifera n'a été capturé (Tableau 1).

En 2000, D. v. virgifera fut observé et des spécimens récoltés dans la Montérégie à Sainte-Barbe, Ormstown, SaintLouis-de-Gonzague, Saint-Etienne, Sainte-Martine ainsi qu'à Saint-Bruno-deMontarville (Fig. 1, Tableau 1).

Diabrotica $v$. virgifera et $D$. barberi abondent dans les champs de maïs cultivés sur des sols argileux lourds et ces deux espèces passent l'hiver au stade oeuf. À cause des conditions climatologiques peu favorables rencontrées durant la saison 2000, l'éclosion et le développement larvaire se sont étalés sur une plus longue période de temps et les adultes émergèrent tard en août. Des adultes ténéraux des deux espèces ont été récoltés aussi tardivement que le 3 octobre 2000.

En Ontario, D. v. virgifera a été rapporté pour la première fois en 1975 (Foott et Timmins 1977). K. Bereza (communication personnelle) et l'auteur principal ont suivi son déplacemnent vers l'est, si bien qu'en 1990, elle était présente à Ottawa, Winchester, Prescott et Cobden (Agriculture Canada 1991). En 1999, des observations dans le maïs, ainsi que du dépistage à l'aide de pièges lumineux et de filets-fauchoirs dans la luzerne, le trèfle et le soja ont confirmé la présence de $D$. $v$. virgifera à l'ouest de la route qui part d'Ottawa et se rend à Iroquois (route 31), à l'exception d'une mention à l'est de la route 138 (Crysler) par J.M. Montpetit (communication personnelle).

Diabrotica v. virgifera cause des dommages similaires mais plus importants que ceux de $D$. barberi. Les larves et les adultes peuvent occasionner une diminution importante des rendements. Les larves qui s'alimentent à partir des radicelles ou creusent des galeries dans les racines primaires du maïs peuvent causer la verse du maïs ou la formation de cols d'oie. Si la regénération des parties endommagées ou la croissance des autres racines est trop lente, une pluie abondante ou un fort vent peuvent augmenter la verse et rendre la récolte plus difficile. $D^{\prime}$ autre part, les adultes des deux espèces s'alimentent du limbe des feuilles réduisant ainsi la photosynthèse ainsi que du pollen sur

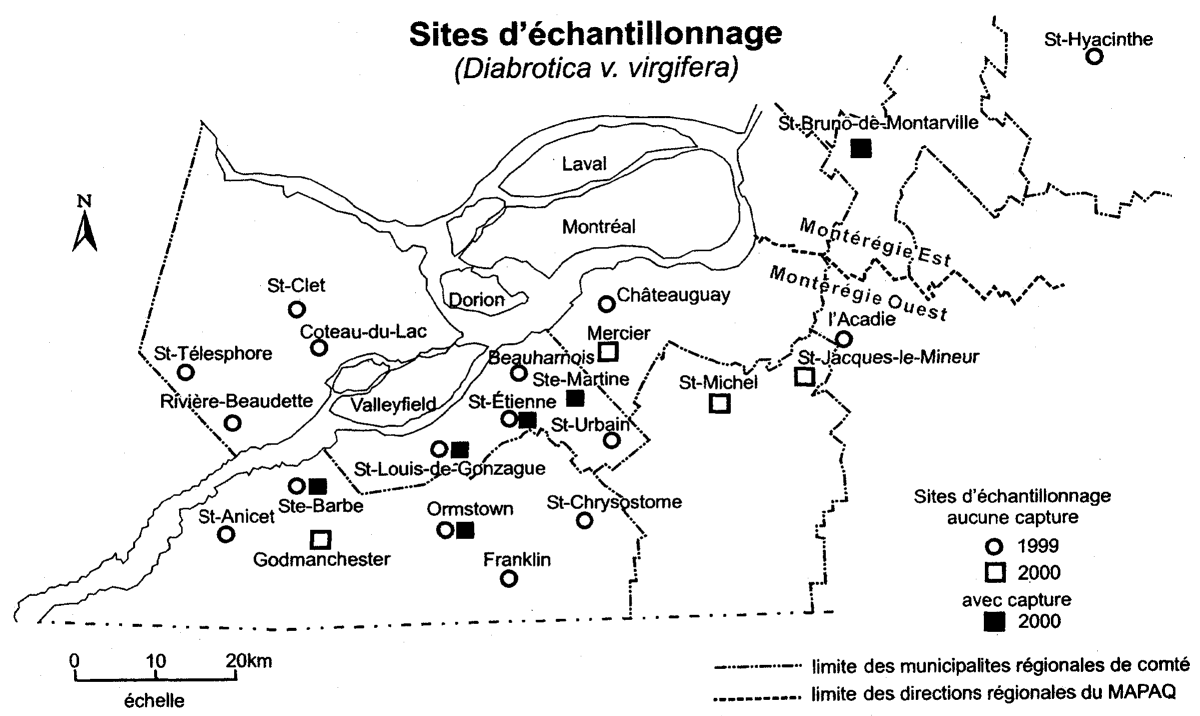

Figure 1 : Sites inventoriés en 1999 et 2000 
Tableau 1. Nombre de Diabrotica capturés à l'aide du filet-fauchoir en 1999 et 2000 dans la luzerne, le trèfle et le soja

\begin{tabular}{|c|c|c|c|c|}
\hline \multirow[b]{2}{*}{ Site } & \multicolumn{3}{|c|}{ Diabrotica } & \multirow{2}{*}{$\begin{array}{l}\text { Nombre total de } \\
\text { coups de filet }\end{array}$} \\
\hline & barberi & virgifera & howardi & \\
\hline \multicolumn{5}{|l|}{1999} \\
\hline Saint-Hyacinthe & 110 & 0 & 1 & 200 \\
\hline Saint-Pie-de-Bagot & 123 & 0 & 1 & 100 \\
\hline Saint-Damase & 71 & 0 & 2 & 200 \\
\hline Saint-Liboire & 31 & 0 & 0 & 100 \\
\hline Sainte-Barbe & 62 & 0 & 0 & 100 \\
\hline Saint-Polycarpe & 338 & 0 & 2 & 200 \\
\hline \multicolumn{5}{|l|}{2000} \\
\hline Saint-Michel & 1 & 0 & 0 & 100 \\
\hline Saint-Jacques-le-Mineur & 43 & 0 & 1 & 100 \\
\hline Saint-Bruno-de-Montarville & 19 & 7 & 0 & 100 \\
\hline Sainte-Barbe & 32 & 1 & 1 & 200 \\
\hline Ormstown & 499 & 1 & 0 & 200 \\
\hline Godmanchester & 61 & 0 & 0 & 100 \\
\hline
\end{tabular}

${ }^{a}$ Coups de filet donnés en septembre

la panicule ou sur les soies de l'épi. Le broutage intensif des soies peut produire des épis non remplis et une diminution du rendement. Les épis endommagés par la pyrale du maïs (Ostrinia nubilalis Hübner) ou les oiseaux attirent les chrysomèles qui se nourrissent de l'amidon des grains. Les adultes deviennent ainsi des vecteurs de maladie, comme la fusariose de l'épi qui diminue la qualité du grain et les rendements.

Aux États-Unis, on a rapporté qu'une

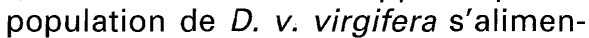
tait sur le maïs et sur le soja (Steffey et al. 1999). Afin de vérifier si ce phénomène existait au Canada, nous avons entrepris en 1999, et répété en 2000 , de placer à la Ferme expérimentale centrale d'Ottawa (ON) des entonnoirs renversés enfoncés dans le sol dans la pépinière de Diabrotica. Cette pépinière mesure $55 \mathrm{~m} \times 100 \mathrm{~m}$ et a été ensemencée avec du maïs depuis 1989. En 1999, une moitié de la pépinière a été semée avec du soja, l'autre avec du maïs et vice-versa en 2000. Durant les deux saisons, des adultes ont été récoltés dans le soja (Tableau 2) confirmant ainsi que $D$. v. virgifera a complété son cycle biologique sur le soja au Canada également. L'examen des racines de soja a permis d'observer la présence de dommages superficiels et des galeries semblables à celles observés sur le maïs.

Tableau 2. Nombre de Diabrotica spp. récoltés à I'aide d'entonnoirs renversés placés dans du soja et du maïs à Ottawa en 1999 et 2000

\begin{tabular}{lccccccc}
\hline & \multicolumn{3}{c}{ Diabrotica } & & \\
\cline { 2 - 5 } & \multicolumn{3}{c}{ barberi } & v. virgifera & & Nombre d'entonnoirs \\
\cline { 2 - 6 } & $1999^{\mathrm{a}}$ & $2000^{\mathrm{b}}$ & $1999^{\mathrm{a}}$ & $2000^{\mathrm{b}}$ & $1999^{\mathrm{a}}$ & $2000^{\mathrm{b}}$ \\
Soja & 7 & 0 & 46 & 2 & 32 & 24 \\
Maïs & 23 & 16 & 25 & 44 & 16 & 32 \\
\hline
\end{tabular}

a Captures du 19 juillet au 31 août 1999.

b Captures du 6 juillet au 1 septembre 2000. 
Bereza (1986), Hartman (1997) et Schaafsma (1991) suggéraient d'effectuer une rotation avec le soja pour contrôler les populations de chrysomèles sur le maïs. Nos observations remettent en question cette pratique de gestion pour les chrysomèles des racines. Puisque nous avons trouvé une population de $D$. $v$. virgifera qui se développe sur le soja, la rotation du maïs au soja risque d'amplifier les problèmes occasionnés par ce ravageur plutôt que de les réduire.

\section{RÉFÉRENCES}

Agriculture Canada. 1991. Rapport de la direction générale de la recherche, Ottawa, Ont. Publication 5252, p. 129.

Bereza, C. 1986. Corn rootworms. OMAFRA, AGDEX $111 / 622,3 \mathrm{p}$.

Campbell, J.M., M.J. Sarazin et D.B. Lyons. 1989. Canadian beetles (Coleoptera) injurious to crops, ornamentals, stored prod- ucts and buildings. Res. Branch Agric. Canada, Publication 1826, p. 85-90.

Foott, W.H. et P.R. Timmins. 1977. Observations on new insect pest of grain corn in Essex county, Ontario. Proc. Entomol. Soc. Ontario $108:$ 49-52.

Hartman, T.R. 1997. Corn rootworms, OMAFRA, revision Agdex 111/622, 3 p.

Hudon, M, G. Boivin, P. Martel, J. Belcourt et C. Ritchot. 1984. Les ravageurs du maïsQuébec. Page 3 in J. Kelleher (éd.), La revue Canadienne des insectes nuisibles aux cultures, vol. 62. Agriculture Canada. Ottawa, Ontario.

O'C. Guibord, M. 1976. La chrysomèle des racines du maïs, Diabrotica longicornis (Say) (Coleoptères: Chrysomelidae), au Québec. Ann. Soc. Entomol. Québec 21 : 49-51.

Schaafsma, A. 1991. Corn rootworms : The obstinate pest that won't go away. Ontario corn producer, Aug.-Sept. 1991, p. 5-6.

Steffey, K.L., M.E. Rice, J. All, D.A. Andow, M.E. Gray et J.W. Van Duyn. 1999. Handbook of corn insects. Entomological Society of America, Maryland, USA. p. 61-68. 